\title{
Fuzzy Logic Based DSR Trust Estimation Routing Protocol for MANET Using Evolutionary Algorithms
}

\author{
Eswaramoorthy VELLINGIRI*, Vinoth Kumar KALIMUTHU, Gopinath SAMYDURAI
}

\begin{abstract}
In MANET attaining consistent routing is a main problem due to several reasons such as lack of static infrastructure, exposed transmission medium, energetic network topology and restricted battery power. These features also create the scheme of direction-finding protocols in MANETs become even more interesting. In this work, a Trust centered routing protocol is suggested, since trust plays a vital role in computing path in mobile ad hoc networks (MANETs). Estimating and computing trust encourages cooperation in mobile ad hoc networks (MANETs). Various present grade systems suddenly estimate the trust by considering any one of the parameters such as energy of node, number of hops and mobility. Estimating trust is an Energetic multi objective optimization problem (EMOPs) typically including many contradictory goals such as lifetime of node, lifetime of link and buffer occupancy proportion which change over time. To solve this multi objective problem, a hybrid Harmony Search Combined with Genetic algorithm and Cuckoo search is used along with reactive method Dynamic Source routing protocol to provide the mobile hosts to find out and sustain routes between the origin node (SN) to the target node (TN). In this work, the performance of the direction-finding practice is assessed using throughput, end to end delay, and load on the network and route detection period.
\end{abstract}

Keywords: Mobile ad hoc network; reactive; reputation; trust

\section{INTRODUCTION}

Mobile Ad hoc Networks (MANET) are frameworks made of autonomous portable hosts which interconnect with all others over wireless associations. The versatile hosts, which are in correspondence scope of every host, are able to straightforwardly convey, yet others need the guide of transitional hosts to transmit their data (Mohammed et al 2007). MANETs are useful without the support of any settled foundation and are totally appropriated. These systems are made exceptionally adaptable and strong by this property.

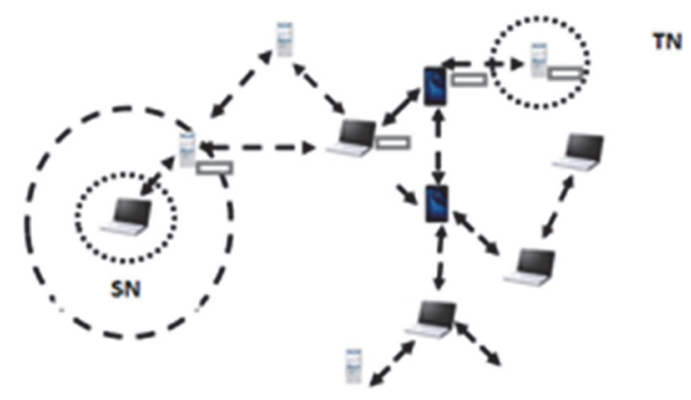

Figure 1 Mobile ad hoc network

In this fashion, the topology of the ad hoc network may vary rapidly and abnormally. However, by the absence of any settled foundation, it gets to be distinctly muddled to abuse the present directing methods for system administrations, and this gives some huge difficulties in giving security to the message, which is not done easily, as the quantity of requests of system security struggle with the prerequisites of portable systems, fundamentally because of the way of the cell phones e.g. low power utilization, low handling load. Fig. 1 demonstrates that MANETs do not rely on any previous infrastructure or base stations.

The routing tactics for MANET remain inherited from conventional systems which can be disciplined to so much criticism as they do not take into account ad-hoc community traits corresponding to mobility and useful resource constraints. Numerous direction finding approaches were suggested. Pre-emptive direction finding protocols invent paths for every pair of hosts by constantly informing the routing tables at constant time intervals with all the hosts in the network. If a host wants to transmit data to any host, the path is available in the direction table. Preemptive direction-finding protocol centered wireless networks have additional overheads within the network as a result of stable updating of route tables; however, origin to target data transfer delay is reduced. However, on demand direction finding protocols found a path to a target only when there is a necessity.

There is no central mechanism to observe the node actions. These features power a constituent node to be careful when cooperating/connecting with other nodes as the conduct of nodes varies with time and environmental conditions. Therefore, creating and measuring behaviour of nodes in the form of trust is vital for safeguarding suitable process of MANET.

Three models, Reputation based, Trust based and Credit based were produced to authorize cooperation and demoralize node trouble making. Utilization of past end client conduct, notoriety, and trust-based plans empower nodes to choose whether to trust different Centres. Inevitably, nodes with high status are offered administrations while those with poor status are disconnected from the system.

\section{RELATED WORKS}

Theodorakopoulos and Baras [1] proposed a mechanism which models the path problem in the mobile ad hoc network on directed graph where nodes represent entities and edges represent trust relations. They used the concept of smearing to assess the trust value or confidence.

Li \& Delgado Frias [2] proposed a scheme, which gives assurance against individual/agreeable getting rowdy hubs. It needs no trust relationship or interruption identification framework to find misconduct. It joins multipath steering and the single way information transmission with a conclusion to end input system. Prior to a hub starting to speak with a goal hub, it picks two hubs 
disjoint ways from a way show one for information transmission and the other for data trade correspondence.

Kejun et al [3] plot concentrated on making trouble connect identification and not getting rowdy hubs which could be an extra to existing source directing conventions like DSR. The framework utilizes uncommon affirmation bundle allowed a settled two jump course or three centres in a bearing inverse to real information activity stream.

Graffi et al [4] proposed a strategy titled Leak Detector which takes a shot at proactive directing utilizing both Watchdog system and powerful affirmations for unique or intriguing hubs. Source hubs have a movement counter for each way demonstrating the measure of transmitted activity on it. Aggregate and Ti fields track general operation sent, and the measure of change a hub I advance regarding the total action. Information is gone through hubs along that way. Suppose N1 passes information to N2. The late first affixed data to the parcels went to hub list field before reporting the measure of information got from $\mathrm{N} 1$ in TN1 zone with respect to reset field $T_{\text {total }}$.

$\mathrm{S}$ Guo \& O Yang [5] presented two dispersed multicast calculations, i.e., fundamental energy productive multicast (FEPM) and disseminated maximum lifetime multicast (DMLM), for a similar advancement issue in MANETs. Dispersed calculations additionally investigate the confined activities to exploit the force sparing advertised by the remote multicast advantage property in mobile networks.

Feng Li \& Jie Wu [6] presented a scheme for the major direction finding mechanisms in MANET proactive and reactive. In both mechanisms the trust and reputation of host are measured such that it reduces the cost, delay and uncertainty.

Sameh \& Milena [7]) proposed thoroughly disseminated notoriety based components for enhanced MANET security. The proposed part acquainted numerous enhancements with existing MANET notoriety plans like appropriate deviation tests, and the versatile close clock went for managing blockage and quick union. Two different centrality measures assess singular trust claims and resolve totalled ones. The proposed technique was planned/worked over AODV and tried in NS-2 amid dark gap assaults in profoundly versatile and inadequate systems. It comes with indicated accomplishment of expanded throughput, diminished postponement and jitter and AODV joining.

M. T. Refaei et al. [8] proposed an adaptive trust management method, which can be able to understand that variations in the activities of the host may be originated from variations in the system conditions.

D. Gutiérrez-Reina et al. [9] suggested a protocol based on topology to calculate the distance between the fixed hosts and speeds of mobile hosts using the Genetic algorithm.

Vazifehdan, et al. [10] suggested two algorithms, in which RMECR addresses three significant issues of ad hoc network: energy efficiency, reliability and extending network lifetime. RMER discovers routes with minimum energy requirement for the exchange of information between source and target.

Manickavelu \& Vaidyanathan [11] have presented a molecule swarm enhancement (PSO) based lifetime expectation calculation (PNLP) for course recuperation in
MANET. Their procedure predicts the lifetime of connection and hub in the accessible data transfer capacity in light of the parameters like the relative versatility of hubs and vitality channel rate, and so forth.

Stan, et al. [12] proposed a proactive OLSR algorithm based technique to find the trust of the mobile host and in turn trust based direction finding algorithm. In this proactive method a dissemination method for trust is designed without any overhead.

G. P. Gupta presented a cuckoo search based algorithm for cluster election in the sensor network by balancing the energy \& the scheme improves the life of network [13].

G. P. Gupta \& S. Jha proposed an improved cuckoo search-based vitality adjusted node grouping convention which utilizes a novel target work for uniform circulation of cluster heads. Likewise, an improved harmony search based directing convention is proposed for steering of the information between cluster heads and the sink [14].

M. Faheem et al. proposed a biopropelled multiobjective developmental directing convention (called MERP) for UWSNs-based applications [15]. The planned directing convention misuses the highlights of the characteristic advancement of the multi-objective genetic calculation so as to give solid and vitality aware data assembling in UWSNs.

E. Fitzgerald et al. introduced optimization plans for increasing the system lifetime, that is, the absolute working time, of a wireless network [16]. Specifically, we have concentrated working on this issue of machine-to-machine correspondence requiring information aggregation and scattering inside the system, where hubs are heterogeneous and may take on several jobs and assignments.

Z. Zhang et al. proposed a hybrid optimization algorithm based on Cuckoo Search (CS) and Differential Evolution (DE) because the single algorithm failed to meet the multiple constraints [17].

I. U. Khan et al. (2020) recommended a method in which the optimal path selection in FANET is done by using nature stimulated procedure named ACO [18]. This work is compared with other protocols such as conventional ACO, DSR, TORA and the outputs show that there is an increase in the energy efficiency and performance.

X. Wang et al. (2020) suggested a routing method which calculates trust entropy and the path with lowest minimum entropy is entered into the routing table [19]. Route selection is made based on the parameters such as number of hops and trust values of hosts. It also reduces the latency and increases the packet delivery ratio.

D. G. Zhang et al. (2020) proposed a technique to overcome the drawback of frequent route rediscovery of the single path protocols [20]. A multipath version of AODV is used and life time of the link, energy model have been accepted. Based on this an ordering mechanism is framed and threshold of every host is calculated. The host having lesser energy threshold is not allowed to take part in the route detection process. Selection of a path is done considering the lifespan of the path and energy threshold, thus improves consumption of energy, PDR and reduces the origin to target delay. 


\section{WORK DESCRIPTION}

\subsection{Estimation of the Life Time of The Node}

In MANET the mobile nodes may be in one of the two states: stationary or dynamic. Because of arbitrary movement of nodes the energy may also get depleted. The lifetime of the host is indirectly proportional to the energy depletion rate (EDR) [10]. The life time of the host is estimated using the following Eq. (1):

$$
N T_{i}=\frac{E R_{i}}{E D R_{i}}
$$

For every $T$ seconds node $i$ reads the instant remaining battery power value $E R_{i}{ }^{0}, E R_{i}{ }^{2 T}, E R_{i}{ }^{3 T}, \ldots \quad E R_{i}{ }^{(n-1) T}, E R_{i}{ }^{n T}$ in every extent $[0, T],[T, 2 T],[2 T, 3 T], \ldots,[(N-1) T, N T]$, $\alpha$ signifies the factor that imitates the relation among $E D R_{i}^{N}$ and $E D R_{i}^{N-1}$ and it has the constant value range of $[0,1]$ and the matching predictable energy depletion rate $E D R_{i}^{N}$ is attained as follows.

$$
\begin{aligned}
& E D R_{i}^{0}=0, N=0 \\
& E D R_{i}^{1}=\frac{E R_{i}^{0}-E R_{i}^{T}}{T}, N=1 \\
& E D R_{i}^{N}=\frac{\alpha\left(E R_{i}^{(N-1)}-E R_{i}^{N T}\right)}{T+(1-\alpha) E R_{i}^{(N-1)}}, N>1
\end{aligned}
$$

\subsection{Estimation of the Link Life Time Between Contiguous Nodes}

The period of link between hosts in the path determines the duration of the path. The period of links between any two adjacent hosts is accessed as follows. Here a random way point mobility model is used and the hosts in the system are placed randomly. The lifetime of the link is accessed based on the placement of nodes on the network and mobility of the participating hosts in the link [22-27].

$L T_{L}=\min \left(L T_{i j}, E R_{i}, E R_{j}\right)$

where $L T_{i j}$ denotes the connection lifetime among the two hosts $i$ and $j . L T_{L}$ denotes the lifetime of the link which is designed as least of value of the remaining energy of the two adjacent mobile nodes and connections lifetime among the two adjacent nodes [7].

Assume that $\Omega$ denotes the set of all nodes in route $R$ and that $\Psi$ is the set of all the connections in route R. Thus, the lifetime $L T_{R}$ of route $R$ can be stated in the following equation.

$$
\begin{aligned}
& L T_{R}=\min \left(L T_{i j}, E R_{i}\right) n_{i} \in \Omega, c_{i} \in \Psi \\
& S_{1}^{2}=S_{0}^{2}+\left[V\left(t_{1}-t_{0}\right)\right]^{2}-S_{0} V\left(t_{1}-t_{0}\right) \cos \theta
\end{aligned}
$$

\subsection{Buffer Occupancy Proportion}

A buffer occupancy proportion signifies how much packets are received at every node. If a node experiences from heavy loads, buffer overflow may happen. All the adjacent nodes pass the packets to the one node, since it is the adjacent node to the target node. Here the node which receives the packet from many nodes may experience buffer overflow as it is getting more additional packets from the neighbours than it can exercise. In direction finding, the next node selection decision is made by considering the remaining buffer space of the node. It is defined that remaining buffer space of a node is buffer occupancy proportion and represents the buffer occupancy proportion of node $\mathrm{i}$ as $B O P_{i}$ [12]. The buffer occupancy proportion $(B O P)$ is computed as:

$$
B O P=\frac{P B_{i}}{E B_{i}}
$$

where present buffer size of the node $i$ at time $t$ is denoted as $P B_{i}$. The Extreme buffer space of node is denoted as $E B_{i}$. Due to the varying processing ratio and no nodes in the transmission range, the data may be prone to invalid because of transmission delay. So, to avoid performance degradation by rapid fluctuations in the assessment, weighted change average (WCA) is used. The decision makers prefer WCA as a component for assessing the situation. In WCA the weights are assigned in such a way that more weightage is assigned for recent data and less weightage is assigned for old data. The time $t$ depends on the welcome message clock. A three-period $W C A$ is utilized as follows.

$$
W C A_{B O P_{I}}=\frac{a_{1} \cdot B O P_{i}^{t-2}+a_{2} \cdot B O P_{i}^{t-1}+a_{3} \cdot B O P_{i}^{t}}{a_{1}+a_{2}+a_{3}}
$$

\section{EVOLUTIONARY COMPUTING ALGORITHMS}

EC strategies have been applied to numerous fields. Three developmental registering techniques are utilized to take care of the optimization of various parameters to discover route in MANET. The techniques are explained as follows.

\subsection{Genetic Algorithm}

The Genetic Algorithm (GA) is a pursuit method dependent on the mechanics of characteristic hereditary qualities and natural selection. GA is an appealing and elective device for tackling complex multimodal improvement issues. Specifically, GA re-enacts the natural procedures that permit the successive generations in a populace to adjust to their surroundings. The alteration methodology is for the most part associated through hereditary legacy from guardians to youngsters and through natural selection. The hereditary calculation object makes sense of which people ought to endure, which should copy, and which should kick the bucket. It also records estimations and decides to what degree the development should proceed.

GA begins with an underlying populace whose components are termed genes. The gene comprises a static amount of factors which are called genes. So as to assess and rank genes in a populace, a fitness function dependent on the target capacity ought to be characterized. Each 
individual is given out a "wellness score" according to how extraordinary an answer for the issue it is. Three administrators must be resolved to build up the absolute structure of the GA procedure: choice, hybrid and transformation administrators.

\subsection{Cuckoo Search Optimization}

CS is an improvement method created by Yang and Deb (2010) built up on the brood parasitism of cuckoo species by laying their eggs in the homes of other host fowls. Cuckoo species do not offer any maternal upkeep for their own future generations. Many winged animal species perceive a cuckoo egg dumped in their own home. If a host fledgling finds the eggs which are not their own, it will either dispose of these remote eggs or essentially surrender its home and manufacture another home elsewhere. So the cuckoo consistently attempts to improve its mimicry of its hosts' eggs relating to the hosts' ability to find strategies for perceiving the parasitic egg [21]. The battle between the host and the parasite is practically equivalent to proceeding with serious endeavour, each attempting to out-endure the other. Parasitic cuckoos that exhibit the biggest measures of egg mimicry are those whose hosts show strange measures of egg dismissal lead.

Each egg in a home signifies an answer and a cuckoo egg indicates another arrangement. The better new arrangement (cuckoo) is supplanted by an answer which isn't excellent in the home. In the most little difficult structure, each home has one egg. Another arrangement is made by demand flight.

\subsection{Harmony Search Algorithm (HSA)}

Harmony search is music centered meta-heuristic optimization process. It was presented by the observation that the point of music is to scan for a perfect state of harmony. This harmony in music is practically equivalent to determine the optimality in an optimization procedure. The search technique in optimization can be compared with a jazz performer's act of spontaneity process. From single viewpoint, the perfectly satisfying agreement is dictated by the complete stylish usual. An artist consistently imagines, creating a bit of music with flawless coordination.

Again, a best resolution for an optimization issue must be the best clarification available to the issue below the specified goals and limited by constraints. The two mentioned methods lead to deliver the best or ideal. Such equalities among two processes can be employed to develop fresh algorithms by taking advantage from each other [14]. Agreement exploration is just such a productive case by changing the individual improvisation procedure into some quantitative guidelines through idealization, also in this way altering the glory and harmony of music into an optimization method via looking for perfect harmony, to be specific, the Harmony Search (HS) or Harmony Search Algorithm (HSA).

\subsection{Hybrid Evolutionary Algorithm Using GA, Cuckoo and Harmony Search}

The recommended hybrid evolutionary procedure calculates the link lifetime; node lifetime and buffer occupancy proportion [17]. The objective function for the hybrid algorithm is given in the following equation.

$$
f(o b j)=\left(w_{1} \cdot N T_{i}\right)+\left(w_{2} \cdot L T_{i}\right)+\left(w_{3} \cdot B O P_{i}\right)
$$

where $w_{1}, w_{2}$ and $w_{3}$ are weightages assigned to each parameter that varies from 0 to 1 . Random selection method is used to assign values between 0 to 1 for $w_{1}, w_{2}$ and $w_{3}$. Here the weightage is selected by spinning the wheel that ranges from 0 to 1 . The primary focus of the objective function is to maximize the lifetime of node and link, also to minimize the buffer occupancy proportion. To optimally access these parameter the algorithm takes inputs like $E R_{i}, E D R_{i}, P B_{i}$ and $E B_{i}$.

Solving energetic optimization problems (EOP) is more challenging since it requires the optimization technique not only to find the optimal solution but also to track the changing optimal solution. Evolutionary algorithms (EA) and swarm intelligence (SI) are decent applicants to solve EOPs as they are stimulated from biological development and self- ordered systems, these systems, if not flexible by nature, they reveal the possible to adjust to ecological changes, which is beneficial in solving optimization problems in dynamic environments like routing in MANETs [18-20].

In the proposed routing algorithm, multiple chromosomes are created by a node using GA and multiple cuckoo eggs are created by a node using CS algorithm. Here the chromosomes and cuckoo eggs represent the possible solution, which means the discovering of the optimal neighbor node by means of assessing the lifetime of link, node and buffer space. Similarly multiple harmonies are created by a node using HS to find the above said parameters of the node. In MANET due to its inevitable characteristics, it is possible to achieve maximum throughput and minimum delay subject to the estimation of the parameters $L T_{i}, N T_{i}$ and $B O P_{i}$.

Pseudo Code for Hybrid Evolutionary Algorithm using GA, CS and HS is listed below:

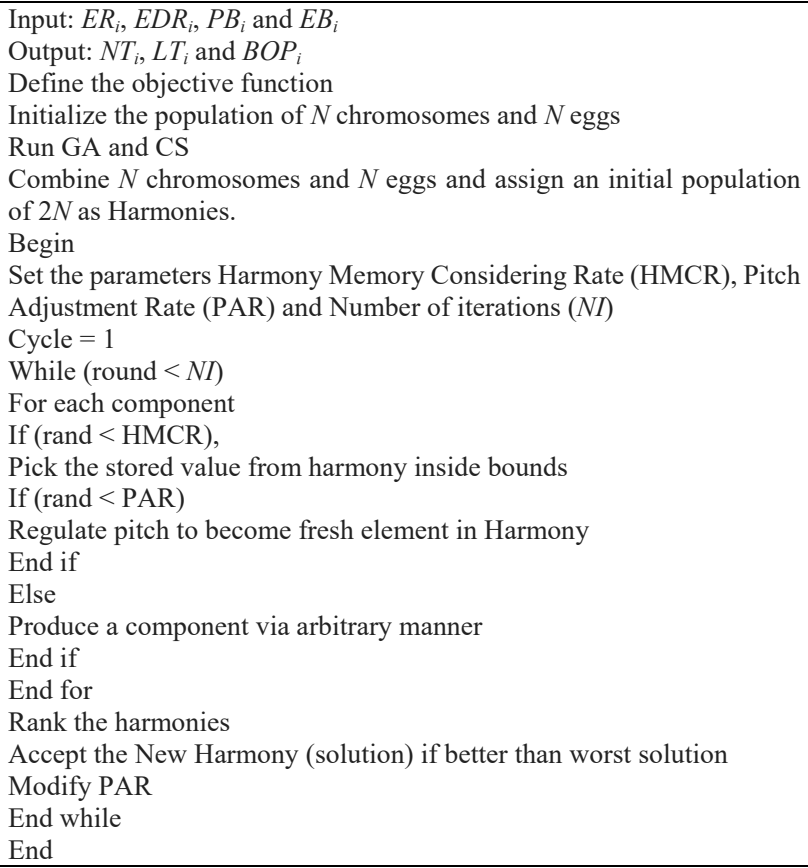


Therefore, a path with a maximum lifetime of the nodes, lifetime of the links and minimum buffer occupancy proportion gets selected and is used for transfer of data packets with a higher probability.

\section{FUZZY BASED DSR TRUST ESTIMATION PROTOCOL}

Fuzzy Logic (FL) is a multiesteemed logic permitting intermediate values to be defined between regular assessments like genuine/false, yes/no, high/low, and so on. Thoughts like rather tall, quick are defined mathematically and prepared by PCs, to apply a more human way of thinking in PC programming. Fuzzy systems are the other options to routine thoughts of enrollment and logic that began in old Greek theory [10]. FL gives a direct way to deal with getting in contact at exact conclusions in perspective of dark, unclear, free, tumultuous, or missing data information.

This process involves fuzzification of parameters $L T_{i}$, $N T_{i}$ and $B O P_{i}$, framing fuzzy logic rules, accumulation of all outputs and defuzzification. The estimation of trust of a mobile node is shown in the figure.

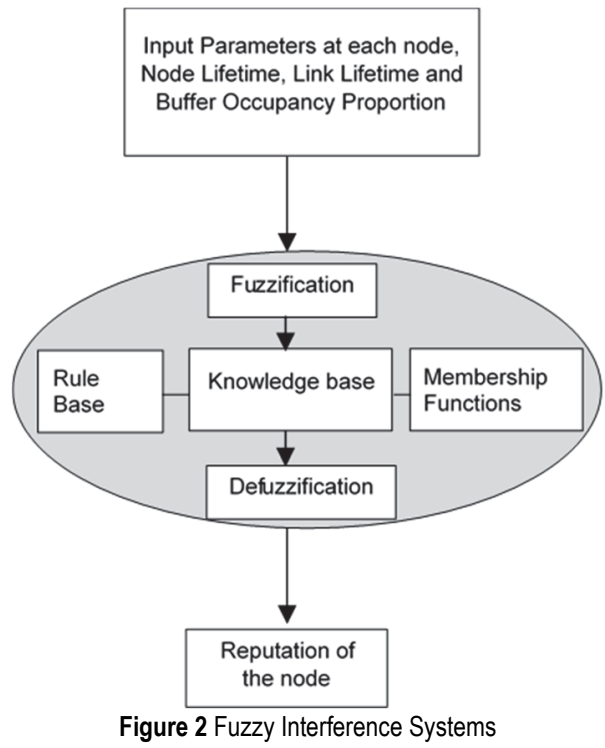

\subsection{Fuzzification}

In this phase, the input parameters are applied to discover the specific input values ranges from o to 1 through the membership functions. There two kind fuzzy inference systems are available, from which Mamdani fuzzy inference systemis used. Several different membership functions can be used on mamdani inference system such as triangular, trapezoid, singleton, sigmoid, Gaussian, etc.Triangular membership function is used and it varies from 0 to 1 in order to fuzzify the input parameters. Based on the input values of the parameters $L T_{i}, N T_{i}$ and $B O P_{i}$ each parameter is classified in to minimum (min), maximum (max) and average (avg).

\subsection{Fuzzy Rule Base}

Fuzzy rule base consists of "if-then" rules, which is used to provide the mapping of input parameters to the output. In this phase, based on the fuzzified input parameters fuzzy rules are framed, it contains different combinations of all the three input parameters $\left(L T_{i}, N T_{i}\right.$ and $\left.B O P_{i}\right)$ and these are mapped to output. The following table displays the sample fuzzy rule.

\begin{tabular}{|c|c|c|c|c|}
\hline \multirow{2}{*}{ Table 1 Fuzzy Rules } \\
\cline { 3 - 5 } & $L T_{i}$ & $\min$ & $\mathrm{avg}$ & $\max$ \\
\cline { 2 - 5 } & $N T_{i}$ & $\max$ & $\min$ & $\mathrm{avg}$ \\
\cline { 2 - 5 } & $B O P_{i}$ & $\max$ & $\mathrm{avg}$ & $\min$ \\
\hline \multicolumn{2}{|c|}{ Output } & LOW & MEDIUM & $\mathrm{HIGH}$ \\
\hline
\end{tabular}

\subsection{Accumulation of all Outputs}

The output of each rule is collected and combined into the output fuzzy set as it contains values ranging from 0 to 1. Also the output fuzzy set consists of number of values equal to the amount of rules framed in the rule book.

\subsection{Defuzzification}

In this phase, the input is output fuzzy set and it is converted into the value that also ranges from 0 to 1 and it is termed fuzzy cost or trust of the mobile node. So the fuzzy cost is mapped to estimating the trust of the mobile node as LOW, MEDIUM and HIGH.

\subsection{Route Discovery}

Path finding allows the source node (SN) to find a path to some other host or target (TN) in the ad hoc network powerfully, regardless of whether straightforwardly available inside remote transmission or through middle nodes. Two sort of directing methodologies utilized in the ad hoc network, reactive and proactive. This work utilizes a reactive conventional Dynamic Source Routing (DSR) which recognizes path at whatever point required. A host (SN) starting a path disclosure communicates a Route Demand (RDEM) packet which might be acquired by those hosts inside the remote transmission scope of it. The RDEM packet recognizes the host, indicated to as the objective of the path location, for which the path is mentioned. In the event that the path finding is effective the SN gets a route response (RRES) packet posting a succession of hops through which it might arrive at the TN. There exist number of paths between the SN and TN. Subsequently the trust of every host will be taken as metric to choose the ideal path. Every host in the system contains the trust or fuzzy outcome (FO).

At the point when a middle host gets a RDEM packet, it computes its FO of taking part in the path as appeared in Fig. 3 and adds it to the FO present on the highest point of heap of the acquired RDEM packet. The subsequent total is then pushed on the stack and afterward RDEM packet is sent to a neighbor host.

Even though the RDEM packet is disseminated in the network, the packets which are reaching the TN contain FO of all the nodes in the path. It will be conveyed to the SN through RRES packet. While RDEM packet traverses 
towards TN, FO of the each node is added and stored in the RDEM packet. When RDEM reaches TN, it will send the RRES consists of the path and FO. The SN will now select the route with highest $\mathrm{FO}$ so that it increases the throughput, minimizes the end to end delay, Load and media access delay. The estimation of fuzzy outcome and route finding process is shown in the below diagram.

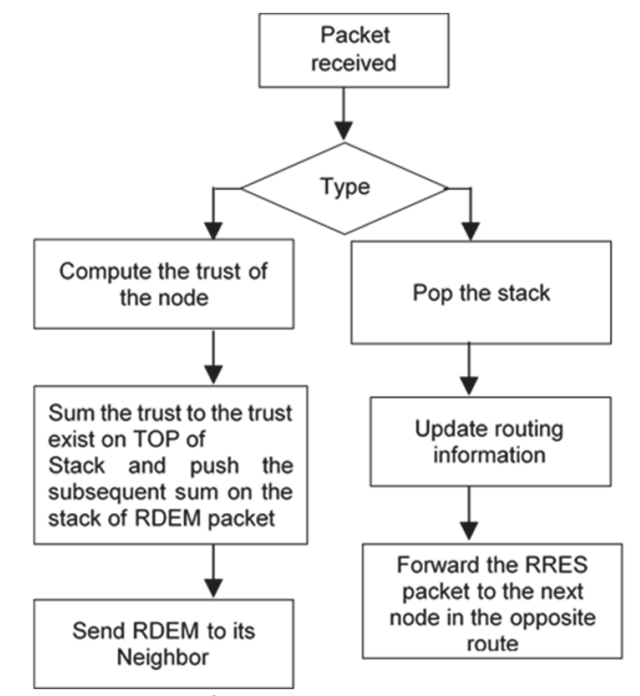

Figure 3 Reaction of transitional nodes while it receives packets

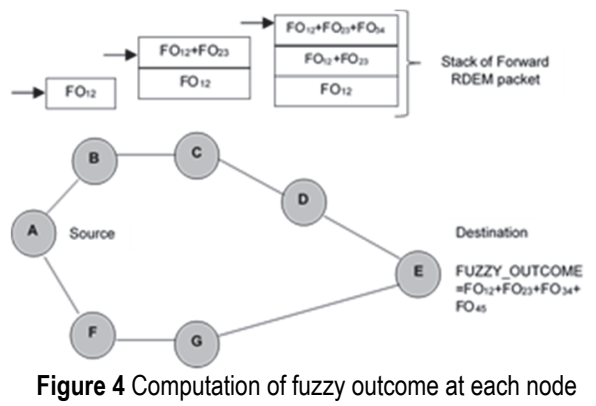

\subsection{Route Maintenance}

This is the stage worried about the routing disappointments, which are caused especially through host versatility. The proposed convention perceives a route disappointment through a missing affirmation. If a host gets a Route Revival Advice (RRA) message for a specific connection, it initially deactivates this connection by setting the FO incentive to 0 . After the disappointment of a connection, the middle host of the route which gets the RRA message will start the backup path to go revelation process by communicating the RDEM message, which is trailed by the path discovery process portrayed previously.

\subsection{Experimental Set Up}

So as to confirm the rightness of the methodology and to envision the presentation in a genuine application, this examination set up an ad hoc network with fifty hosts circulated over $1000 \mathrm{~m} \times 1000 \mathrm{~m}$ space inside the simulation stage all through 600 seconds. An irregular waypoint portability model was utilized: each host selfassertively chose a balance and pushed toward that area with speed beginning from essentially on $0 \mathrm{~m} / \mathrm{s}$ to $50 \mathrm{~m} / \mathrm{s}$. When the host arrived at that position, it got fixed for a programmable respite time; at that point it chose another position and rehashed the strategy. This work played out the simulation with variable stop times $0,60,120,180$, $240,300,360,420,480,540$ and $600 \mathrm{sec}$. The reproduction parameters utilized in the investigation are given in Tab. 2 .

Table 2 Simulation parameters

\begin{tabular}{|c|c|}
\hline Simulation Software & OPNET \\
\hline Mobility Model & Random Waypoint \\
\hline Physical Characteristics & $\begin{array}{c}\text { Extended Rate PHY (802.11 } \\
\text { g) }\end{array}$ \\
\hline Imitation Time & 600 seconds \\
\hline Data Rate / bps & $2 \mathrm{Mbps}$ \\
\hline Imitation Area & $1000 \mathrm{~m} \times 1000 \mathrm{~m}$ \\
\hline Transmit Power / W & -9005 \\
\hline Packet Reception-Power Threshold / \\
dBm
\end{tabular}

A portable host is thought to be fixed with delay time of 600 seconds. A host moves endlessly with zero second stop time. Each host highlights radio spread scope of a hundred and fifty meters, and the information rate is 2 Mbps. The source-target sets are portrayed arbitrarily over the network.

\section{RESULTS AND DISCUSSION}

The outcomes present the performance of the protocol in the accompanying tables and charts. The suggested protocol is contrasted with AODV, DSRand PNLP.

Tab. 3 gives the outcome of throughput, and it is seen that the throughput ascends with the development in stop time for the proposed work. The throughput of the proposed convention improves by $3.8 \%$ when contrasted with AODV when host stop time is $60 \mathrm{sec}$. Likewise, the throughput of the proposed convention improves by $5.6 \%$ and $1.25 \%$ when contrasted with DSR and PNLP when host delay time is $540 \mathrm{sec}$, as appeared in Fig. 5.

\begin{tabular}{|c|c|c|c|c|}
\hline Time / sec & AODV & DSR & PNLP & Proposed \\
\hline 0 & 0 & 0 & 0 & 0 \\
\hline 60 & 2287.70 & 1655.30 & 2196.17 & 2379.20 \\
\hline 120 & 4837.40 & 2529.30 & 4643.94 & 5079.30 \\
\hline 180 & 12543.00 & 7114.60 & 12041.63 & 12794.00 \\
\hline 240 & 11602.00 & 7270.70 & 11137.54 & 12530.00 \\
\hline 300 & 12602.00 & 9045.00 & 12098.31 & 13106.00 \\
\hline 360 & 17244.00 & 11963.00 & 16554.24 & 18624.00 \\
\hline 420 & 11934.00 & 6387.50 & 11456.87 & 12292.00 \\
\hline 480 & 14808.00 & 6618.60 & 14215.22 & 15548.00 \\
\hline 540 & 11317.00 & 6252.80 & 10864.74 & 11996.00 \\
\hline 600 & \#N/A & \#N/A & \#N/A & \#N/A \\
\hline
\end{tabular}

The end to end delay of the recommended routing protocol is less by $4.5 \%$ while equated to AODV and by $4.5 \%$ while equated to DSR when node pause time is 60 sec. Likewise, the end to end delay is less by $3.5 \%$ while 
matched to AODV and by $1.6 \%$ and $1.2 \%$ when equated to DSR and PNLP respectively when node pause time is 540 sec as revealed in Fig. 6.

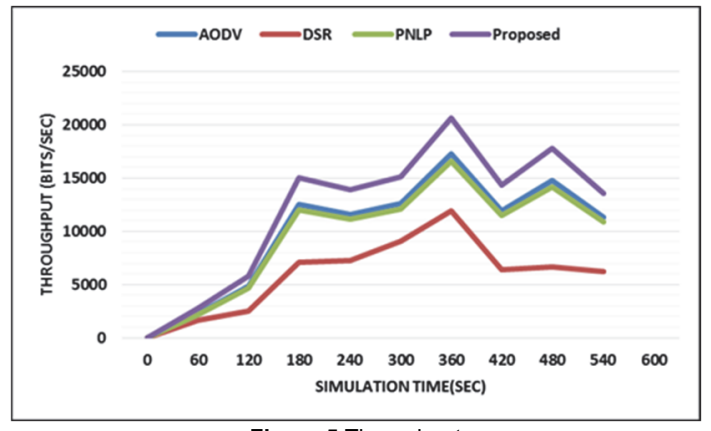

Figure 5 Throughput

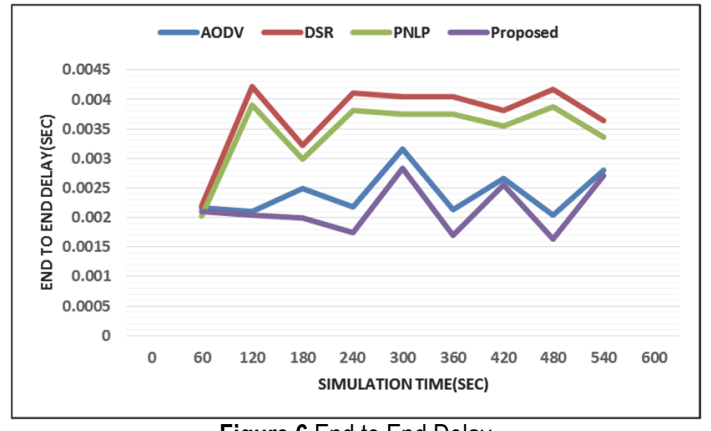

Figure 6 End to End Delay

It is observed that the load decreases for the planned routing scheme. The load of the recommended directionfinding protocol is less by $2.4 \%$ when compared to AODV and by $4 \%$ when compared to DSR when node pause time is $60 \mathrm{sec}$. Similarly, the load of the proposed routing protocol is less by $5.3 \%$ when compared to AODV and by $3 \%$ \& $1.2 \%$ when compared to DSR and PNLP repectively when node pause time is $540 \mathrm{sec}$ as shown in Fig. 7.

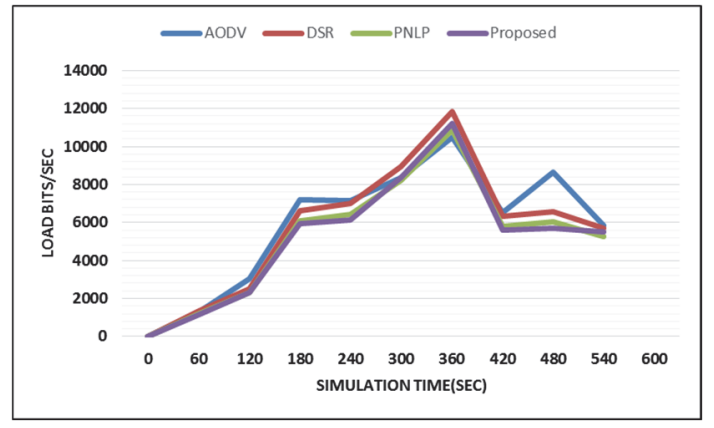

Figure 7 Load

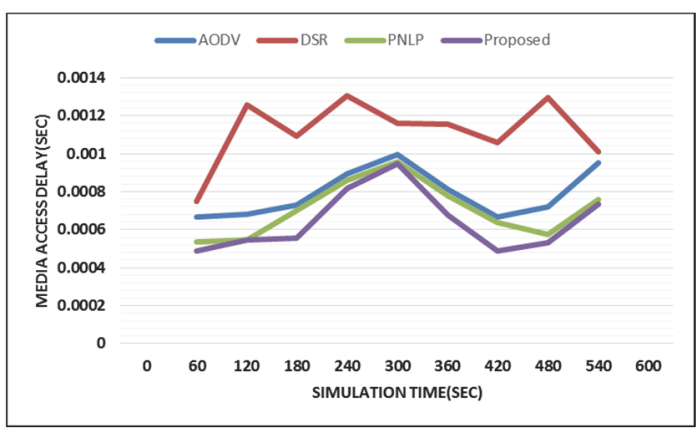

Figure 8 Media Access Delay
It is perceived that the media access delay declines with the rise in pause time for the suggested directionfinding protocol. The media access delay of the projected direction-finding protocol is less when related to AODV, DSR and PNLP as shown in Fig. 8.

It is observed that the route discovery time for the planned protocol is very small when related to standard AODV and DSR. Fig. 9 shows the same. Though the total route cache sent is high for the proposed routing, the total packet dropped is considerably less than that of AODV and DSR. Fig. 10 shows the same.

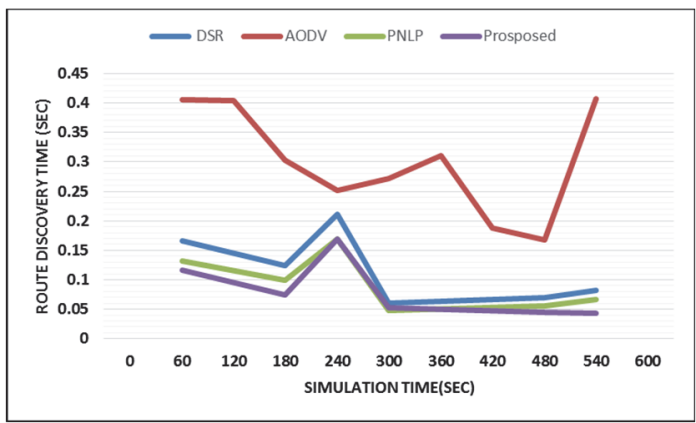

Figure 9 Route Discovery Time

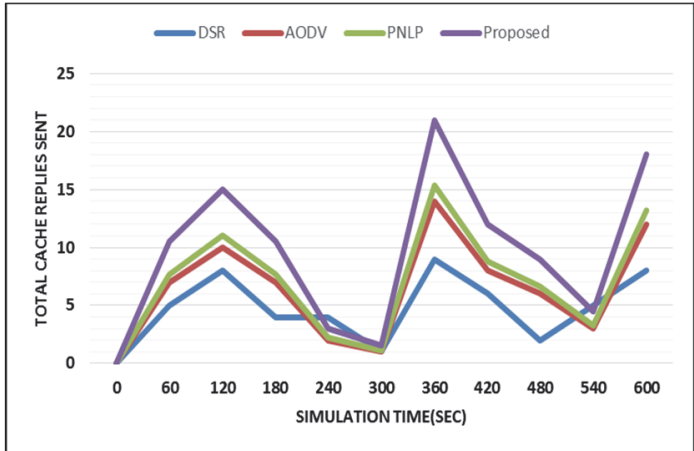

Figure 10 Total cache replies sent

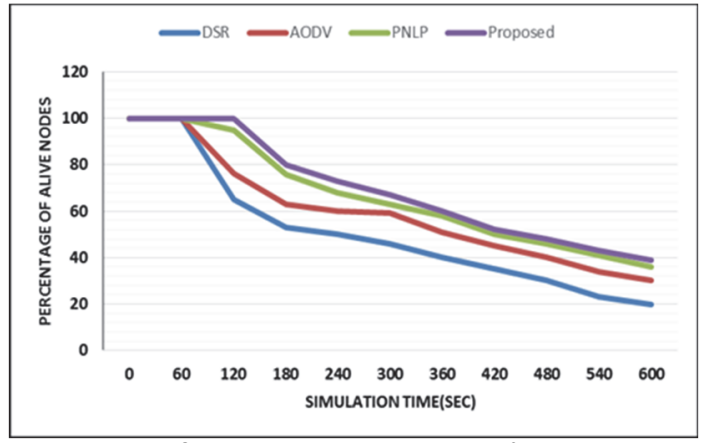

Figure 11 Simulation time Vs Percentage of alive nodes

The percentage of active nodes against the simulation time is displayed in Fig. 11. The initial energy of the nodes is considered as 10.2 joules. The simulation is executed for $600 \mathrm{sec}$. The percentage of nodes alive at the end of simulation is $40 \%$ for the proposed method. This is due to the fact that new solutions are informed about the finest result, to quicken the native exploration.

\section{CONCLUSION}

In this research, Fuzzy Logic based DSR Trust estimation routing protocol for MANET using Evolutionary algorithms is proposed. In the route detection 
process, the trust of the node is calculated using fuzzy logic. For computing trust the life period of link, node and buffer occupancy proportion are taken as parameters and they are estimated using GA, Cuckoo and Harmony Search. Based on the computed trust values optimum path is selected. In the route maintenance process, alternate route is used whenever there is failure in the route. This work mainly reduces the number of route rediscovery. Thus, it improves the lifetime of route in turn it increases the throughput, reduces the delay, load and route discovery time. Additional work should be done for high volume information like video conferencing and high density mobile nodes.

\section{REFERENCES}

[1] Theodorakopoulos, G. \& Baras, J. S. (2006). On Trust Models and Trust Evaluation Metrics for Ad Hoc Networks. IEEE journal on selected areas in communications, 24(2), 31-38. https://doi.org/10.1109/JSAC.2005.861390

[2] Li, Z. \& Delgado Frias, J. G. (2007). MARS: Misbehavior Detection in AdHoc Networks. Global Telecommunications Conference, 941-945.

[3] Kejun, L., Jing, D., Pramod, K. V., \& Kashyap, B. (2007). An Acknowledgment-based Approach for the Detection of Routing Misbehavior in MANETs. IEEE Transactions on Mobile Computing, 6(5), 536-550. https://doi.org/10.1109/TMC.2007.1036

[4] Graffi, K., Mogre, P. S., Hollick, M., \& Steinmetz, R .(2007). Detection of Colluding Misbehaving Nodes in Mobile Ad Hoc and Wireless Mesh Networks. Global Telecommunications Conference, 5097-5101. https://doi.org/10.1109/GLOCOM.2007.966

[5] Guo, S. \& Yang, O. (2008). Maximizing Multicast Communication Lifetime in Wireless Mobile Ad Hoc Networks. IEEE Transactions On Vehicular Technology, 57(4), 2414-2425. https://doi.org/10.1109/TVT.2007.912607

[6] Feng, L. \& Jie, W. (2010). Uncertainty Modeling and Reduction in MANETs. IEEE transactions on mobile computing, 9(7), 1035-1048. https://doi.org/10.1109/TMC.2010.44

[7] Sameh, Z. \& Milena, R. (2010). Reputation-based security protocol for MANETS in highly mobile disconnection-prone environments. Proceedings of the seventh international conference on wireless on-demand network systems and services, 161-167.

[8] Refaei, M. T., DaSilva, L. A., Eltoweissy, M., \& Nadeem, T. (2010). Adaptation of Reputation Management Systems to Dynamic Network Conditions in Ad Hoc Networks. IEEE Transactions on Computers, 59(5), 707-719. https://doi.org/10.1109/TC.2010.34

[9] Gutiérrez Reina, D., Marín, S. L. T., Johnson, P., \& Barrero, F. (2012). An evolutionary computation approach for designing mobile ad hoc networks. Expert Systems with Applications, 32, 6838-6845. https://doi.org/10.1016/j.eswa.2012.01.012

[10] Manickavelu, D. \& Vaidyanathan, R. U. (2014). Particle swarm optimization (PSO)-based node and link lifetime prediction algorithm for route recovery in MANET. EURASIP Journal on Wireless Communications and Networking, 1-10. https://doi.org/10.1186/1687-1499-2014-107

[11] Vazifehdan, J., Prasad, R. V., \& Niemegeers, I. (2014). Energy-Efficient Reliable Routing Considering Residual Energy In Wireless Ad Hoc Networks. IEEE Transactions On Mobile Computing, 13(2), 434-447. https://doi.org/10.1109/TMC.2013.7
[12] Tan, S., Li, X., \& Dong, Q. (2015). Trust Based Mechanism for Securing OLSR based MANET. Ad hoc Networks, 30(7), 84-98. https://doi.org/10.1016/j.adhoc.2015.03.004

[13] Gupta, G. P. (2018). Improved Cuckoo search based Clustering Protocol for Wireless Sensor networks. Procedia Computer Science, 125(7), 234-240. https://doi.org/10.1016/j.procs.2017.12.032

[14] Gupta, G. P. \& Jha, S. (2018). Integrated clustering and routing protocol for wireless sensor networks using Cuckoo and Harmony Search based metaheuristic techniques. Engineering Applications of Artificial Intelligence, 68, 101109. https://doi.org/10.1016/j.engappai.2017.11.003

[15] Faheem, M., Ngadi, M. A., \& Gungor, V. C. (2019). Energy efficient multi-objective evolutionary routing scheme for reliable data gathering in Internet of underwater acoustic sensor networks. Ad hoc Networks, 93, 1-18. https://doi.org/10.1016/j.adhoc.2019.101912

[16] Fitzgerald, E., Pióro, M., \& Tomaszewski, A. (2019). Network lifetime maximization in wireless mesh networks for machine-to-machine communication. Ad hoc Networks, 95, 1-12. https://doi.org/10.1016/j.adhoc.2019.101987

[17] Zhang, Z., Ding, S., \& Jia, W. (2019). A hybrid optimization algorithm based on cuckoo search and differential evolution for solving constrained engineering problems. Engineering Applications of Artificial Intelligence, 85, 254-268. https://doi.org/10.1016/j.engappai.2019.06.017

[18] Inam, U. K., Ijaz, M. Q., Muhammad, A. A., Tanweer, A. C., \& Syed, B. H. S. (2020). Smart IoT Control-Based Nature Inspired Energy Efficient Routing Protocol for Flying Ad Hoc Network (FANET). Special Section On Evolving Technologies In Energy Storage Systems For Energy Systems Applications, 8, 56371-56378. https://doi.org/10.1109/ACCESS.2020.2981531

[19] Wang, X., Zhang, P., Du, Y., \& Qi, M. (2020). Trust Routing Protocol Based on Cloud-Based Fuzzy Petri Net and Trust Entropy for Mobile Ad hoc Network. Special Section On Evolving Technologies In Energy Storage Systems For Energy Systems Applications, 8, 47675-47693. https://doi.org/10.1109/ACCESS.2020.2978143

[20] Zhang, D. G., Chen, L., Zhang, J., Chen, J., Zhang, T., Tang, Y. M., \& Qiu, J. N. (2020). A Multi-Path Routing Protocol Based on Link Lifetime and Energy Consumption Prediction for Mobile Edge Computing. Special Section On Evolving Technologies In Energy Storage Systems For Energy Systems Applications, 8, 69058-69071. https://doi.org/10.1109/ACCESS.2020.2986078

[21] Yang, X. S. \& Deb, S. (2010). Engineering optimization by cuckoo search. International journal of mathematical modeling and numerical optimization, 1(4), 330-343. https://doi.org/10.1504//JMMNO.2010.035430

[22] Wang, X. \& Li, J. (2015). Improving the network lifetime of MANETs through cooperative MAC protocol design. IEEE Trans. Parallel Distrib. Syst., 26(4), 1010-1020. https://doi.org/10.1109/TPDS.2013.110

[23] Akande, D. O. \& Salleh, M. F. M. (2019). A Network Lifetime Extension-Aware Cooperative MAC Protocol for MANETs With Optimized Power Control. IEEE Access, 7, 18546-18557. https://doi.org/10.1109/ACCESS.2019.2895342

[24] Sudheera, K. L. K., Maode, M., \& Chong, P. H. J.(2019). Link Stability Based Optimized Routing Framework for Software Defined Vehicular Networks. IEEE Transactions On Vehicular Technology, 68(3), 2934-2945. https://doi.org/10.1109/TVT.2019.2895274

[25] Ma, Y. \& Shu, J. (2019). ONs Link Prediction Method Based on BRNN. IEEE Access, 7(1), 18576-185795. https://doi.org/10.1109/ACCESS.2019.2961243

[26] Gune, I., Gunduz, O. S., \& Çataltepe, Z. (2016). Link prediction using time series of neighborhood-based node similarity scores. Data Mining Knowl. Discovery, 30(1), 147-180. https://doi.org/10.1007/s10618-015-0407-0 
[27] Ozcan, A. \& Oguducu, S. G. (2018). Link prediction in evolving heterogeneous networks using the NARX neural networks. Knowl. Inf. Syst., 55(2), 333-360. https://doi.org/10.1007/s10115-017-1073-x

\section{Contact information:}

Eswaramoorthy VELLINGIRI, PhD, Assistant Professor

(Corresponding author)

Department of Information Technology,

Bannari Amman Institute of Technology,

Sathyamangalam, TamilNadu, India

E-mail: eswarinfotech@gmail.com

Vinoth Kumar KALIMUTHU, PhD, Associate Professor

Department of Electronics and Communication Engineering

SSM Institute of Engineering and Technology,

Dindigul, Tamil Nadu, India

E-mail: vinodkumaran87@gmail.com

Gopinath SAMYDURAI, PhD, Associate Professor

Electronics and Communication Engineering,

Karpagam Institute of Technology,

Coimbatore, Tamil Nadu, India

E-mail: gopi.vasudev@gmail.com 